\title{
Plasma phospholipid fatty acid status of 5-year-old children in the Seychelles Child Development Nutrition Study
}

\author{
A. J. McAfee ${ }^{1}$, M. S. Barnes ${ }^{1}$, J. J. Strain ${ }^{1}$, P. W. Davidson ${ }^{2}$, M. P. Bonham ${ }^{1}$, E. M. McSorley ${ }^{1}$, \\ S. W. Thurston ${ }^{2}$, J. M. W. Wallace ${ }^{1}$, C. F. Shamlaye ${ }^{3}$, G. Watson ${ }^{2}$, G. J. Myers ${ }^{2}$ and T. W. Clarkson ${ }^{2}$ \\ ${ }^{1}$ Northern Ireland Centre for Food and Health, University of Ulster, Coleraine, BT52 1SA, UK, ${ }^{2}$ School of Medicine and \\ Dentistry, University of Rochester, USA, ${ }^{3}$ Ministry of Health, Seychelles and ${ }^{4}$ Cornell University, USA and ${ }^{5}$ The Johns \\ Hopkins Bloomberg School of Public Health, USA
}

The long-chain n-3 PUFA (LCn-3PUFA), eicosapentaenoic $\left(\mathrm{C}_{20: 5}\right)$ and DHA $\left(\mathrm{C}_{22: 6}\right)$, supplied by dietary intake of fish, exert a range of benefits to human health, with DHA having an important role in infant visual and brain development ${ }^{(1)}$. We have previously reported that prenatal status of LCn-3PUFA, as measured by the geometric mean of the 28 -week and delivery values during pregnancy, was positively associated with a measure of infant development at 9 months of age ${ }^{(2)}$. In the present study, the plasma phospholipid fatty acid status of children at 5 years old was investigated within the Seychelles Child Development Study.

In this longitudinal cohort of mother child pairs recruited from the Republic of Seychelles, blood samples were collected from the children $\left(n\right.$ 227), with mothers providing informed consent. From plasma aliquots previously stored at $-80^{\circ} \mathrm{C}$, total lipid was extracted according to a modified method of Folch et al. ${ }^{(3)}$ and the technique of solid phase extraction was used to obtain plasma phospholipids. Fatty acid methyl esters were prepared with boron trifluoride (Sigma-Aldrich Co. Ltd) and analysed using an Agilent 5975C GC-MS (Agilent, UK). Summary statistics were generated for fatty acid status and an independent samples $t$-test was used to compare means of boys and girls (SPSS v. 17.0).

Table 1. Plasma phospholipid fatty acid (presented as $\mathrm{mg} / \mathrm{ml}$ ) status of 5-year-old Seychellois children

\begin{tabular}{|c|c|c|c|c|c|c|c|}
\hline \multirow[b]{2}{*}{ Myristic acid } & \multicolumn{2}{|c|}{ ALL ( $n$ 227) } & \multicolumn{2}{|c|}{ Boys (n 117) } & \multicolumn{2}{|c|}{ Girls (n 110) } & \multirow{2}{*}{$\frac{P^{*}}{\mathrm{NS}}$} \\
\hline & 0.008 & $(0.004,0.020)$ & 0.006 & $(0.004,0.018)$ & 0.008 & $(0.004,0.021)$ & \\
\hline Palmitic acid & 0.250 & $(0.189,0.336)$ & 0.250 & $(0.192,0.331)$ & 0.255 & $(0.186,0.3490)$ & NS \\
\hline Stearic acid & 0.112 & $(0.084,0.151)$ & 0.112 & $(0.086,0.146)$ & 0.113 & $(0.083,0.152)$ & NS \\
\hline Oleic acid & 0.044 & $(0.029,0.067)$ & 0.044 & $(0.028,0.068)$ & 0.045 & $(0.030,0.067)$ & NS \\
\hline Linoleic acid & 0.106 & $(0.078,0.147)$ & 0.106 & $(0.079,0.144)$ & 0.106 & $(0.076,0.154)$ & NS \\
\hline AA & 0.044 & $(0.030,0.061)$ & 0.044 & $(0.029,0.062)$ & 0.046 & $(0.030,0.061)$ & NS \\
\hline EPA & 0.004 & $(0.002,0.008)$ & 0.004 & $(0.002,0.008)$ & 0.004 & $(0.002,0.008)$ & NS \\
\hline DHA & 0.030 & $(0.020,0.044)$ & 0.030 & $(0.020,0.044)$ & 0.030 & $(0.020,0.044)$ & NS \\
\hline
\end{tabular}

Results are median (5th, 95th percentiles) and are presented as $\mathrm{mg} / \mathrm{ml}$ plasma.

$* P$ values refer to differences between boys and girls, where NS is non significant.

AA, arachidonic acid.

The mean age of the children at this time-point was 5.6 years old and there was no significant difference between boys and girls (data not shown). Table 1 shows the plasma phospholipid status of the total number of children $(n 227)$ and of the boys ( $n$ 117) and girls ( $n$ 110). The median EPA and DHA concentrations of the children were 0.004 and $0.03 \mathrm{mg} / \mathrm{ml}$, respectively. Sex differences in LCn-3 PUFA status have previously been reported in adults, with women having significantly higher DHA status ${ }^{(4)}$; however, in this study, no significant differences in fatty acid status were found between boys and girls aged 5 years.

In conclusion, concentrations of LCn-3PUFA as measured in plasma phospholipids within a group of children residing in Seychelles are similar to concentrations reported for adults from a different population ${ }^{(5)}$; however, this is the first time that data on fatty acid status in this age group and from this region have been reported. No sex differences were found for fatty acid status in this group. These data will be used as potential modifiers of the relationship between maternal methylmercury exposure from fish consumption and developmental outcomes in the offspring.

This work was supported by grant nos. PO1 ES01248, ROI ES008442, 2 T 32 ES007271, PO ES01247, 5-RO1-ES010219 and 2-T32-ES007271 from the US National Institute of Environmental Health Sciences, National Institutes of Health, the Government of Seychelles and by the EU through its Sixth Framework Programme for RTD (contract no. FOOD-CT-2006-016253).

1. Ruxton CHS, Calder PC, Reed SC et al. (2005) Nutr Res Rev 18, 113-129.

2. Strain JJ, Davidson PW, Bonham MP et al. (2008) Neurotoxicology 29, 776-782.

3. Folch J, Lees M \& Sloane Stanley GH (1956) J Biol Chem 226, 497-509.

4. Crowe FL, Skeaff CM, Green TJ et al. (2008) Br J Nutr 99, 168-174.

5. de Groot RHM, Hornstra G, van Houwelingen AC et al. (2004) Am J Clin Nutr 79, 251-260. 